\title{
Influence of the Reaction Heat on the Linear Stability Spectra of Steady Detonation in the Kinetic Frame
}

\author{
Filipe Carvalho* and Ana Jacinta Soares ${ }^{\dagger}$ \\ * Departamento de Ciências Exactas, Viana do Castelo, Portugal \\ ${ }^{\dagger}$ Departamento de Matemática e Aplica-Żes, Universidade do Minho, Braga, Portugal
}

\begin{abstract}
The structure and linear stability of the one-dimensional steady overdriven detonation wave supported by a chemical reaction of type $A+A \rightleftharpoons B+B$ are examined in the frame of the Boltzmann equation extended to chemically reacting gases. The structure of the steady wave solution is determined solving the system of the Rankine-Hugoniot conditions supplemented with the rate law, in a chemical regime which corresponds to the early stage of the reaction process. The response of such steady wave solution to one-dimensional disturbances is investigated using a normal mode linear analysis which leads to an initial value problem for the state variable disturbances in the reaction zone. Some results are obtained numerically in order to describe the stability spectra of the steady solution. The emphasis of the present study is on the influence of the reaction heat on the linear stability spectra.
\end{abstract}

Keywords: Boltzmann equation, Chemical reactions, Steady detonation waves, Stability.

PACS: $51.10 .+\mathrm{y}$ 47.70.Pq 82.33.Vx 47.20.-k

\section{INTRODUCTION}

The one-dimensional steady detonation wave is commonly described in literature [1], [2] using the reactive hydrodynamic equations and related Rankine-Hugoniot jump conditions. The configuration of the steady wave solution is qualitatively described by the ZND model [2] and consists in a plane non-reactive shock propagating with constant velocity, followed by a finite reaction zone where the chemical reaction takes place. This rather standard approach has been used in classical fluid mechanics [3]-[6] more recently, in the framework of the kinetic theory for chemically reactive system [7]-[10]. In particular, in the recent paper [9] by the present authors, the dynamics of the steady detonation in a binary mixture undergoing a chemical reaction of type $A+A \rightleftharpoons B+B$ has been characterized with the main purpose of analysing the effects of the reaction heat on the detonation wave solution. The structure of the one-dimensional steady detonation wave solution has been characterized in that paper resorting to a different closure procedure of the reactive hydrodynamic equations. In fact, instead of the usual equilibrium Maxwellian distribution function, the hydrodynamic closure is based on the non-equilibrium solution of the Boltzmann equation obtained in paper [11] by means of the Chapmann-Enskog method in a chemical regime proper of the early stage of the reaction process, when the elastic time scale is smaller than the reactive one.

On the other hand, experimental studies [1], [2], [12] reveal that the ZND detonation solution tends to be structurally unstable since a small rear perturbation of the wave front can affect the steady character of the state variables in the reaction zone and the detonation solution does not admit anymore a steady configuration. The stability of detonation waves and subsequent evolution of the instabilities in the reaction zone constitutes a relevant research topic in detonation theory, after the pioneering studies conducted by Erpenbeck [13], based on a Laplace transform approach, and many further contributions, see for example papers [4]-[6], [14] based on a normal mode analysis combined with a numerical shooting technique. At the kinetic level, in the frame of the Boltzmann equation extended to chemically reacting gases, the linear stability of steady detonation waves has been formulated for the first time in paper [15], where a rather complete stability picture is shown with reference to the Hydrogen-Oxygen system. Starting from this kinetic approach, the linear stability of the steady detonation solution characterized in paper [9] is here investigated, integrating numerically the ODE's for the steady state disturbances, between the detonation shock and the reaction equilibrium state. Some results on both the steady detonation wave structure and its linear stability are discussed and the numerical technique used to perform the stability simulations is described. 


\section{PRELIMINARIES ON THE REACTIVE KINETIC MODEL}

Consider a binary mixture of constituents $A$ and $B$, with the same mass $m$ and binding energies $E_{A}$ and $E_{B}$, respectively, undergoing binary elastic collisions and inelastic interactions with chemical reaction of type $A+A \rightleftharpoons B+B$. At the kinetic level, the mixture is described by the reactive Boltzmann equations of paper [11], namely

$$
\frac{\partial f_{\alpha}}{\partial t}+c_{i}^{\alpha} \frac{\partial f_{\alpha}}{\partial x_{i}}=\sum_{\beta=A}^{B} \mathcal{Q}_{\alpha \beta}^{E}+\mathcal{Q}_{\alpha}^{R}, \quad \alpha=A, B
$$

where $\mathcal{Q}_{\alpha \beta}^{E}$ and $\mathcal{Q}_{\alpha}^{R}$ are the elastic and reactive collision terms, respectively, whose explicit expressions are omitted here for sake of brevity. In the above cited paper [11], a non-equilibrium solution of the previous Eqs. (1) has been explicitly obtained as a small perturbation of Maxwellian distribution $f_{\alpha}^{M}$, using the asymptotic expansion procedure of Chapman-Enskog in a chemical regime for which the reaction process is close to its initial stage,

$$
f_{\alpha}^{(0)}=f_{\alpha}^{M}\left[1+\omega\left(\frac{15}{8}-\frac{5 m\left(c_{\alpha}-v\right)^{2}}{4 k T}+\frac{m^{2}\left(c_{\alpha}-v\right)^{4}}{8 k^{2} T^{2}}\right)\right],
$$

where $k$ the Boltzmann constant, $v$ and $T$ the mean velocity and temperature of the mixture, and $\omega$ is given by

$$
\omega=x_{A}^{2}\left(\frac{d}{d_{r}}\right)^{2} \frac{Q_{R}^{\star}}{8}\left[\left(1+2 Q_{R}^{\star}-3 Q_{R}^{\star} \varepsilon_{A}^{\star}-Q_{R}^{\star 2}+\varepsilon_{A}^{\star}-2 \varepsilon_{A}^{\star 2}\right) e^{-\mathcal{A}^{\star}}\left(1-Q_{R}^{\star}-Q_{R}^{\star} \varepsilon_{A}^{\star}+\varepsilon^{\star}-2 \varepsilon_{A}^{\star 2}\right)\right] e^{-\varepsilon_{A}^{\star}} .
$$

Above, $x_{A}=n_{A} / n$ is the concentration of the $A$-constituent, $d$ and $d_{r}$ are the elastic and reactive diameters, $\varepsilon_{A}^{\star}$ and $\mathcal{A}^{\star}=\mathcal{A} / k T$ the activation energy and the affinity of the forward reaction in units of $k T$, and $Q_{R}^{\star}=Q_{R} / k T$ is the reaction heat in units of $k T$. Moreover, $Q_{R}=2\left(E_{B}-E_{A}\right)$, so that $Q_{R}>0$ when the forward reaction is endothermic whereas $Q_{R}<0$ when it is exothermic. The solution given by Eqs. (2-3) exhibit an appreciable influence of the reaction heat as well as a small contribution of the affinity, and has been obtained adopting elastic cross sections of hard-sphere type and reactive step cross sections with activation energy [11].

At the macroscopic level, the mixture is described by the governing balance equations for the number densities $n_{\alpha}$ of the constituents, mean velocity $v_{i}$ and temperature $T$ of the mixture,

$$
n_{\alpha}=\int f_{\alpha} d c_{\alpha}, \quad v_{i}=\frac{1}{n} \sum_{\alpha=A}^{B} \int c_{i}^{\alpha} f_{\alpha} d c_{\alpha}, \quad T=\frac{m}{3 k n} \sum_{\alpha=A}^{B} \int\left(c_{\alpha}-v\right)^{2} f_{\alpha} d c_{\alpha},
$$

whose time-space evolution, in one-space dimension, is defined by

$$
\begin{aligned}
& \frac{\partial n_{\alpha}}{\partial t}+\frac{\partial n_{\alpha}}{\partial x_{i}}\left(n_{\alpha} v_{i}+n_{\alpha} u_{i}^{\alpha}\right)=\tau_{\alpha}, \\
& \frac{\partial}{\partial t}\left(\frac{3}{2} n k T+\sum_{\alpha=A}^{B} n_{\alpha} E_{\alpha}+\frac{1}{2} \rho v^{2}\right)+\frac{\partial}{\partial x_{i}}\left\{v_{i}\right)+\frac{\partial}{\partial x_{i}}\left(p_{i j}+\rho v_{i} v_{j}\right)=0 \\
& \left.q_{i}+p_{i j} v_{j}+\left(\frac{3}{2} n k T+\sum_{\alpha=A}^{B} n_{\alpha} E_{\alpha}+\frac{1}{2} \rho v^{2}\right) v_{i}\right\}=0 .
\end{aligned}
$$

Above, $u_{i}^{\alpha}, n, \rho, p_{i j}, q_{i}$ define the diffusion velocity of the $\alpha$-constituent, the number density, mass density, pressure tensor and heat flux of the mixture. Moreover, the term $\tau_{\alpha}$ on the r.h.s. of Eq. $(5)_{1}$ is the reaction rate, given by

$$
\tau_{A}=-4 n_{A}^{2} d_{r}^{2} \sqrt{\frac{\pi k T}{m}} e^{-\varepsilon_{A}^{\star}}\left[1+\varepsilon_{A}^{\star}+\frac{x_{A}^{2}}{128}\left(\frac{d}{d_{r}}\right)^{2} Q_{R}^{\star}\left(1+Q_{R}^{\star}+Q_{R}^{\star} \varepsilon_{A}^{\star}+\varepsilon_{A}^{\star}-2 \varepsilon_{A}^{\star 2}\right)\left(4 \varepsilon_{A}^{\star 3}-8 \varepsilon_{A}^{\star 2}-\varepsilon_{A}^{\star}-1\right) e^{-\varepsilon_{A}^{\star}}\right],
$$

which has been obtained through the closure process of the balance Eqs. (5-6), using the non-equilibrium distribution $f_{\alpha}^{(0)}$ given by expressions (2-3).

\section{STEADY DETONATION SOLUTION}

The main aspects of the steady detonation modelling proposed by the authors in paper [9] are recalled here with the aim of investigating the linear stability of the one-dimensional steady detonation solution characterized in that paper. 
With reference to the ZND idealized description of the detonation wave [2], the steady detonation wave consists in a plane non-reactive shock followed by a finite reaction zone where the chemical reaction takes place. The shock is located at $x=x_{0}$, and the reaction zone remains from $x_{0}$ to $x_{F}$. The state just behind the shock is the Von Neumann state, where the chemical reaction is triggered, and the one located at $x_{F}$ is the final state, where the chemical reaction reaches the equilibrium. Ahead of the shock front, that is for $x>x_{0}$, the gas is in its initial state and the chemical reaction is not yet initiated. Assuming that the shock wave propagates from left to the right with constant velocity $D$ along the $x$-direction, one first introduces the normalized steady variable $x_{s}=(x-D t) / D t_{c}, t_{c}=\sqrt{\frac{m}{\pi k T^{+}}} /\left(4 n^{+} d^{2}\right)$, where the superscript + refers to the initial state. For sake of simplicity, the normalized steady variable $x_{s}$ is still denoted with the plane symbol $x$. The structure of the steady detonation solution of the hydrodynamic equations (5-6) can be determined using the following jump Rankine Hugoniot conditions

$$
\begin{aligned}
n_{B}\left(n_{A}\right) & =\frac{\left(n_{B}^{+}+n_{A}^{+}\right) D}{D-v}-n_{A}, \quad T\left(n_{A}\right)=\frac{(D-v)\left(\rho^{+} D v+n^{+} k T^{+}\right)}{n^{+} k D}, \\
v\left(n_{A}\right) & =\frac{2 Q_{R}^{*} n_{A}+3 \rho^{+} D^{2}-5 n^{+} k T^{+}+\sqrt{\left(2 Q_{R}^{*} n_{A}+3 \rho^{+} D^{2}-5 n^{+} k T^{+}\right)^{2}-32 \rho^{+} Q_{R}^{*} D^{2}\left(n_{A}-n_{A}^{+}\right)}}{8 \rho^{+} D},
\end{aligned}
$$

together with the rate equation describing the advancement of the chemical reaction in the reaction zone, namely

$$
\frac{d n_{A}}{d x}=\frac{D t_{c} \tau_{A}}{v-D+n_{A} \frac{d v}{d n_{A}}} .
$$

Equations (8-10), with $D$ as parameter, characterize any arbitrary state $\left(n_{A}(x), n_{B}(x), v(x), T(x)\right)$ within the reaction zone, say for $x \in\left[x_{0}, x_{F}\right]$, in dependence of the initial state $\left(n_{A}^{+}, n_{B}^{+}, 0, T^{+}\right)$. In particular, the von Neumann state just ahead the shock, where the chemical reaction is not yet initiated, can be characterized by Eqs. (8-9) together with a further jump condition of Rankine Hugoniot type, obtained from Eq. (10) by integration acrossing the shock wave. This problem has been numerically solved in paper [9] for both exothermic and endothermic chemical reactions, in the case of an overdriven detonation wave, that is a detonation wave wich propagates with velocity greater than the ChapmanJouguet one and thus, once initiated, does not need any external support to sustain its further evolution. Figure 1 shows some representative steady detonation profiles for the mixture pressure, assuming the following kinetic and thermodynamical input parameters $D=1700 \mathrm{~ms}^{-1}, n_{A}^{+}=0.35 \mathrm{~mol} / \mathrm{l}, n_{B}^{+}=0 \mathrm{~mol} / \mathrm{l}, \mathrm{m}=0.01 \mathrm{Kg} / \mathrm{mol}, T^{+}=298.15 \mathrm{~K}$, $E_{A}=2400 K$ and $\varepsilon_{A}^{\star}=6$. The pictures show that the steady detonation solution is a reactive rarefaction wave for an exothermic reaction and a reactive compression wave for an endothermic reaction.
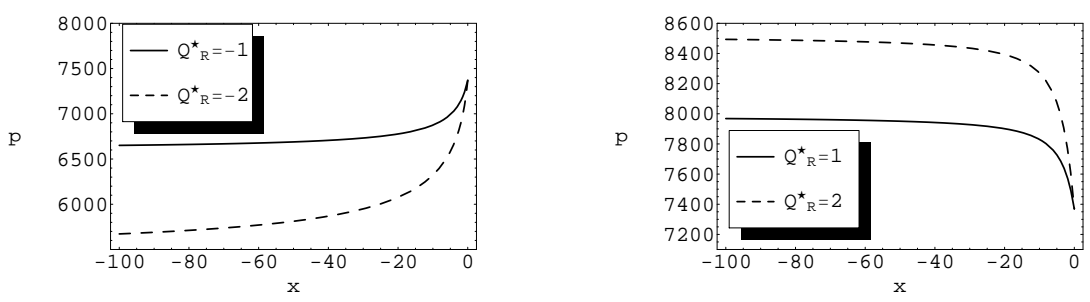

FIGURE 1. Left: Pressure profile for an exothermic chemical reaction with $Q_{R}^{\star}=-1$ (solid line) and $Q_{R}^{\star}=-2$ (dashed line). Right: Pressure profile for an endothermic chemical reaction with $Q_{R}^{\star}=1$ (solid line) and $Q_{R}^{\star}=2$ (dashed line).

\section{LINEAR STABILITY ANALYSIS}

A normal mode linear stability analysis is developed here for the one-dimensional steady overdriven detonation solution characterized in terms of Eqs. (8-10), as described in the previous section. The main purpose is to investigate the response of the steady structure to small rear boundary perturbations which are instantaneousnly assigned and induce a deviation on the planar shock wave. This distortion gives rise to small perturbations on the state variables which propagate in the reaction zone and can either grow or decay in time. The stability of the steady solution is determined by the evolution of those small perturbations in the reaction zone The mathematical analog is defined by the stability equations derived from the one-space dimensional version of the hydrodynamic equations (5-6), through a linearization around the steady solution assuming an exponential time-dependence for the perturbations. 
First, let introduce a more convenient coordinate system, say $t_{a}=t / t_{c}, y=x / D t_{c}$, into the hydrodynamic equations in one-space dimension. For sake of simplicity, relabel the new time coordinate $t_{a}$ with the previous symbol $t$. The following step consists in transforming the resulting equations to the perturbed shock attached frame. At this end, let introduce the shock front displacement from the unperturbed position, $\widetilde{\psi}(t)$, so that the perturbed shock is located at $\psi(t)=D t+\widetilde{\psi}(t)$ and its velocity is $D(t)=D+\widetilde{\psi}^{\prime}(t)$. Let consider then the wave coordinate, $x=y-\psi(t)$, which measures the distance from the perturbed shock and observe that the instantaneous position of the perturbed shock wave is $x=0$ in the new shock-attached coordinate system. The corresponding transformed equations are omitted here. The next step consists in the linearization of the transformed hydrodynamic equations around the steady state, assuming a normal mode expansion for the state variables $n_{A}, n_{B}, v$ and $p$. Intoducing the state vector $z$ defined by $z=\left[\begin{array}{llll}n_{A} & n_{B} & v & p\end{array}\right]^{T}$, the expansions are assumed in the form

$$
z(x, t)=z^{*}(x)+e^{a t} \bar{z}(x), \quad \psi(t)=\bar{\psi} e^{a t}, \quad a, \bar{\psi} \in \mathbb{C},
$$

where $z^{*}(x)$ represents the steady solution and $\bar{z}(x)$ the unknown space disturbances, with $\bar{z} \in \mathbb{C}$. Moreover, $\bar{\psi}$ is a perturbation parameter and $a=\alpha+i \beta$, with $\alpha$ being the perturbation growth rate and $\beta$ the perturbation frequency. The linearization of the transformed governing equations in the perturbed shock frame leads to the stability equations,

$$
\begin{aligned}
& D a \bar{n}_{\alpha}+\left(v^{*}-D\right) \frac{d \bar{n}_{\alpha}}{d x}+\frac{n_{\alpha}^{*}}{d x}(\bar{v}-D a)+\frac{d v^{*}}{d x} \bar{n}_{\alpha}+n_{\alpha}^{*} \frac{d \bar{v}}{d x}=\bar{\tau}_{\alpha}, \quad \alpha=A, B, \\
& \rho^{*} D a \bar{v}+\frac{d \bar{p}}{d x}+\rho^{*} \frac{d v^{*}}{d x}(\bar{v}-D a)+\left(v^{*}-D\right) \frac{d v^{*}}{d x} \bar{\rho}+\rho^{*}\left(v^{*}-D\right) \frac{d \bar{v}}{d x}=0, \\
& D a \bar{p}+\frac{5}{3}\left(p^{*} \frac{d \bar{v}}{d x}+\bar{p} \frac{d v^{*}}{d x}\right)+\left(v^{*}-D\right) \frac{d \bar{p}}{d x}+(\bar{v}-D a) \frac{d p^{*}}{d x}=\frac{Q_{R}^{*} D t_{c} \bar{\tau}_{A}}{3} .
\end{aligned}
$$

In these equations, $\bar{\tau}_{A}$ and $\bar{\tau}_{B}=-\bar{\tau}_{A}$ denote the linearized reaction rates whose form reads

$$
\bar{\tau}_{A}=-4 d_{r}^{2} \sqrt{\frac{\pi k}{m}} e^{-\varepsilon^{\star}}\left[\left(2 n_{A}^{*} \bar{n}_{A} \sqrt{T^{*}}+\frac{\bar{p}+\frac{\bar{n}}{n^{*}} p^{*}}{2 n^{*} k \sqrt{T^{*}}} n_{A}^{* 2}\right)\left(1+\varepsilon^{\star}+\Gamma x_{A} *^{2}\right)+2 \sqrt{T^{*}} \frac{n_{A}^{*}}{n^{*}}\left(-n_{A}^{*} \bar{n}_{B}+n_{B}^{*} \bar{n}_{A}\right)\right],
$$

where $\Gamma=\frac{1}{128}\left(\frac{d}{d_{r}}\right)^{2} Q_{R}^{\star}\left(1+Q_{R}^{\star}+Q_{R}^{\star} \varepsilon_{A}^{\star}+\varepsilon_{A}^{\star}-2 \varepsilon_{A}^{\star 2}\right)\left(4 \varepsilon_{A}^{\star 3}-8 \varepsilon_{A}^{\star 2}-\varepsilon_{A}^{\star}-1\right) e^{-\varepsilon_{A}^{\star}}$. Equations (12-14) govern the dynamics of the perturbations $\bar{z}(x)$ in the reaction zone and have to be integrated from the perturbed shock position $x=0$ to the equilibrium final state $x=x_{F}$. The initial conditions to be joined to these ordinary differential equations connect the value of the disturbances at the von Neuman state to their zero value ahead the perturbed shock. They are provided by the Rankine-Hugoniot relations (8-9) together with the further jump condition for the von Neuman state obtained as explained in the previous section. After applying the coordinate transformations and related normalizations introduced above to the jump conditions at the von Neuman state, the desired initial conditions are obtained in the form

$$
\begin{aligned}
& \bar{n}_{\alpha}(0)=\frac{\left(n_{\alpha}^{*}-n_{\alpha}^{+}\right) a-n_{\alpha}^{*} \bar{v}(0)}{v^{*}-D}, \quad \alpha=A, B, \\
& \bar{v}(0)=\frac{3 \rho^{+} v^{* 2}+\frac{3}{2}\left(p^{*}-p^{+}\right)-\frac{3}{2} D \rho^{+} v^{*}+2 E_{A} n^{+}+Q_{R}^{*} n_{B}^{+}}{-\rho^{*}\left(v^{*}-D\right)^{2}+\frac{5}{2} p^{*}} a, \\
& \bar{p}(0)=-\rho^{+} a v^{*}-\left(v^{*}-D\right) \rho^{*} \bar{v}(0) .
\end{aligned}
$$

The stability equations (12-14), as well as the related initial conditions (16-18), have been deduced introducing the normalization $\bar{w}=\bar{z} / \bar{\psi}$ of the state variables, which avoids the dependence of the the complex parameter $\bar{\psi}$. They constitute a set of eight real equations in the eight unknowns $\operatorname{Re} \bar{w}$ and $\operatorname{Im} \bar{w}$, which is not closed since the parameter $a$ is involved. The necessary closure condition, which gives the dispersion relation for the normal modes (11), is the radiation condition usually adopted in the literature of the detonation stability [15], [16]. This condition states that the inherent instability of the detonation wave solution results exclusively from the interplay between the leading shock and the reaction zone and can not be affected by further disturbances traveling towards the shock from a great distance from the reaction zone. Thus the closure condition is assigned at the equilibrium final state in the form

$$
\bar{v}\left(x_{F}\right)+a=\frac{-1}{\gamma \rho_{e q}^{*} c_{e q}^{*}} \bar{p}\left(x_{F}\right)
$$


where $\gamma$ is the ratio of specific heats, $c_{e q}^{*}$ and $\rho_{e q}^{*}$ the isentropic sound speed and gas density at equilibrium.

The linear stability problem of the steady detonation is formulated in terms of the complex disturbances $\bar{z}(x)$ and perturbation parameter $a$, by means of the ordinary differential equations (12-14) with initial conditions (16-18) and closure condition (19). For a given set of thermodynamical and chemical parameters specifying the structure of the steady detonation wave solution, the disturbances $\bar{z}(x)$ and perturbation parameter $a$ are determined numerically. To do this, a trial value of $a$ in a fixed bounded domain $\mathcal{R}$ of the complex plane is considered and then Eqs. (12-14) are integrated in the reaction zone $\left.] 0, x_{F}\right]$. For a given steady detonation solution, an arbitrary value of $a$ does not satisfy the closure condition (19) and thus it does not produce a solution of the satbility problem. To overcome this difficulty, the residual function $H(a)$, defined from the closure condition by

$$
H(a)=\left|\bar{v}\left(x_{F}\right)+a+\frac{1}{\gamma \rho_{e q}^{*} c_{e q}^{*}} \bar{p}\left(x_{F}\right)\right|, \quad a \in \mathcal{R},
$$

is estimated at each trial value of $a$, and only those solutions $\bar{z}(x)$ obtained for values of $a$ for which the residual function vanish within a given tolerance are accepted. In order to implement the numerical scheme to solve the stability problem, it is convenient to approximate the location of the zeros of $H(a)$ and find a confidence domain $\mathcal{R}$ in the complex plane containing, at least, one zero of the residual function. The search of such a domain requires a rather sophisticated technique which is explained in detail in the extended version of the present work [17]. When the confidence domain is supplied and the zero of $H(a)$ is found in that domain, the numerical scheme provides the entire stability solution, that is the perturbations $\bar{z}(x)$ and the perturbation parameter $a$.

Some simulations have been done using the numerical procedure referred above, in order to describe the stability spectra of the steady detonation solution. The results are shown in the next section.

\section{RESULTS AND DISCUSSION}

The response of the steady detonation solution to the rear boundary perturbations, as well as the influence of the reaction heat on the stability spectra, is investigated solving numerically the stability problem formulated in terms of the perturbation parameter $a$ and perturbations $\bar{z}$ by the Eqs. (12-19). For the simulations, a rectangular region $\mathcal{R}$ such that $0<\operatorname{Re} a<0.02$ and $0.001<\operatorname{Im} a<0.1$ is considered in the right complex half-plane. For the following kinetic and thermodynamical input parameters $D=1700 \mathrm{~ms}^{-1}, n_{A}^{+}=0.35 \mathrm{~mol} / \mathrm{l}, n_{B}^{+}=0 \mathrm{~mol} / \mathrm{l}, \mathrm{m}=0.01 \mathrm{Kg} / \mathrm{mol}$, $T^{+}=298.15 K, E_{A}=2400 K$ and $\varepsilon_{A}^{\star}=6$. The results on the stability problem are in dimensionless form. The influence of the reaction heat on the stability spectra is investigated by considering the reaction heat varying in a given domain. In the present paper the analysis is restricted to the range $0.75 \leq Q_{R}^{*} \leq 1$, corresponding to endothermic chemical reactions. When the reaction heat increases the number of zeros of the residual function $H(a)$ in the right complex half-plane decreases. In the left Frame of Figure 2, the migration in the complex plane of the zero with lower imaginary part, corresponding to that zero with lower perturbation frequency, is shown. The results shown in the other pictures have been obtained for $Q_{R}^{*}=1$. A three-dimensional plot of $H(a)$ in a sub-region of $\mathcal{R}$ is shown in the right Frame of Figure 2, where the location of one zero is evidentiated. Typical unstable profiles are exhibited in Figure 3 for $\operatorname{Re} a=10^{-5}, \operatorname{Im} a=6,0419 \times 10^{-2}$, where the real part of the pressure perturbation, $\operatorname{Re} \bar{p}$, is represented in the reaction zone. Frame 1 exhibits a complete profile whereas Frame 2 shows a detailed profile in the vicinity of the von Neuman state, As expected, the profile shows that the perturbation is greater at the von Neuman state and tends to decay when the distance from the perturbed shock increases. The instability spectrum is complemented in Frames 3, 4 of Figure 3 , where the time evolution of the real part of the total pressure perturbation $\widehat{p}=e^{a t} \bar{p}$, at the von Neuman state, is represented. Frame 3 evidentiates the increasing time behaviour of the perturbation, and Frame 4 shows a detailed representation.

The real parts of the other state variables perturbation as well as the corresponding imaginary parts, show an analogous behaviour. See paper [9] for more detailed results.

\section{ACKNOWLEDGMENTS}

The paper is partially supported by FCT Phd Grant SFRH/BD/28795/2006, by the Research Centre of Mathematics of the University of Minho through the FCT Pluriannual Funding Program and by Project FCT-PTDC/MAT/68615/2006. 

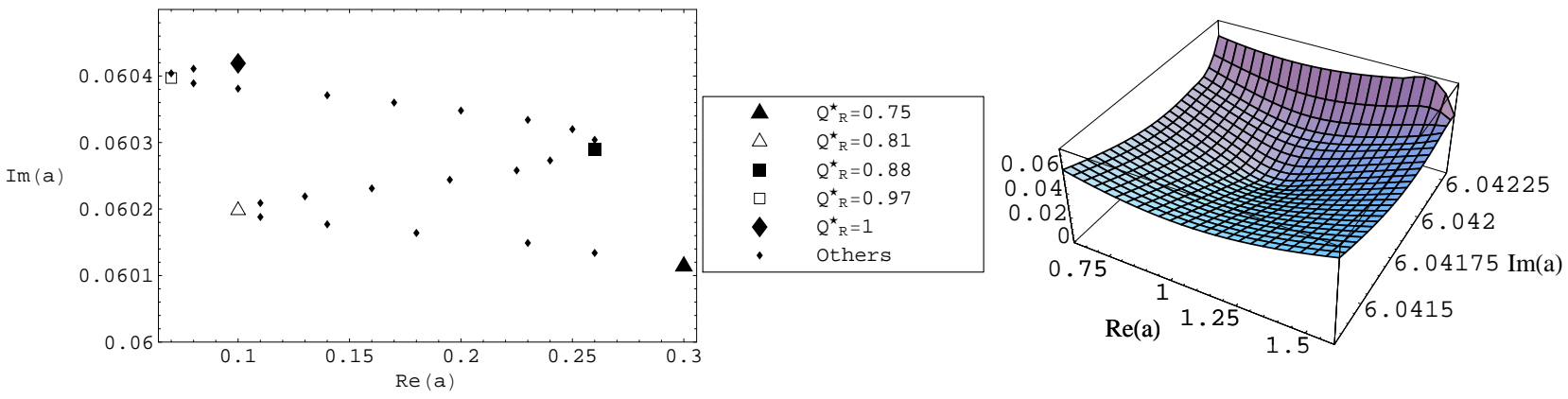

FIGURE 2. Instability spectrum. Left: Migration in the complex plane of the zero $a$ of the residual function $H(a)$ with lower imaginary part. The reaction heat varies from 0.75 to 1 ( $\operatorname{Re} a$ is scaled by the factor $10^{4}$ and $\operatorname{Im} a$ is not scaled). Right: Threedimensional plot of $H(a)$ in a sub-region of $\mathcal{R}$, for $Q_{R}^{*}=1\left(\operatorname{Re} a\right.$ is scaled by the factor $10^{5}$ and $\operatorname{Im} a$ is scaled by $10^{2}$ ).
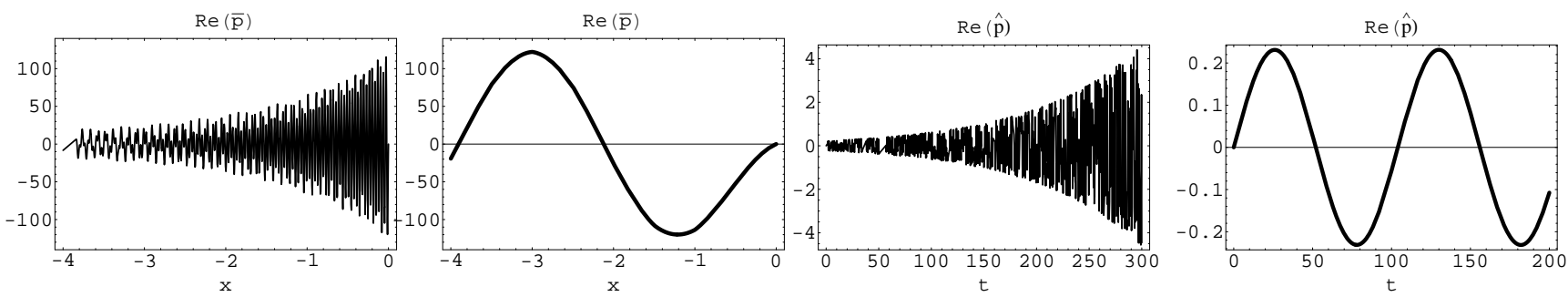

FIGURE 3. Instability spectrum for $Q_{R}^{*}=1, \operatorname{Re} a=10^{-5}, \operatorname{Im} a=6,0419 \times 10^{-2}$. Frame 1: Profile of $\operatorname{Re} \bar{p}$ in the reaction zone $(x$ scaled by the factor $\left.10^{3}\right)$. Frame 2: Detail of $\operatorname{Re} \bar{p}$ at the beginning of the reaction zone ( $x$ scaled by 10$)$. Frame 3: Time evolution of Re $\widehat{p}$ at the von Neuman state $\left(t\right.$ scaled by $\left.10^{3}\right)$. Frame 4 : Detail of $\operatorname{Re} \widehat{p}(t$ not scaled $)$.

\section{REFERENCES}

1. W. Fickett and W. C. Davis, Detonation, Theory and Experiment (University of California, Berkeley) 1979.

2. W. Fickett, Introduction to Detonation Theory (University of California, Berkeley) 1986.

3. J. B. Bdzil and D. S. Stewart, Phys. Fluids, 1, 1261-1267 (1989).

4. A. Kasimov and D. S. Stewart, Phys. Fluids, 16, 3566-3578 (2004).

5. S. Yungster and K. Radhakrishnam, Shock Waves, 14, 61-72 (2005).

6. A. Kasimov and D. S. Stewart, SIAM J. Appl. Math., 66, 384-407 (2005).

7. M. Pandolfi Bianchi and A. J. Soares, Phys. Fluids 8 17-34 (1996).

8. F. Conforto, R. Monaco, F. Schürrer and I. Ziegler, J. Phys. A: Math. Gen. 36 5381-5398 (2003).

9. F. Carvalho and A. J. Soares, "Steady detonation wave solutions under the reaction heat effect", in WASCOM 2009, edited by A. M. Greco et.al. World Scientific Proceedings, Singapore, 2010, pp. 87-92.

10. M. Bisi, M. Groppi and G. Spiga KRM 3 17-34 (2010).

11. G. M. Kremer and A. J. Soares, J. Stat. Mech., P12003 1-16 (2007).

12. R. A. Strehlow, Astro. Acta, 15 345-357 (1970).

13. J. J. Erpenbeck, Phys. Fluids, 5, 604-614 (1962).

14. H. I. Lee and D. S. Stewart, J. Fluid Mech. 216, 103-132 (1990).

15. M. Pandolfi Bianchi and A. J. Soares, Linear Stability of Steady Detonation in Extended Kinetic Theory J. Diff. Equations and Applic. (accepted).

16. A. Kasimov and D. S. Stewart, J. Fluid Mech., 466, 179-203 (2002).

17. F. Carvalho and A. J. Soares (extended paper in preparation). 